\section{SP3-12 PREDICTORS OF 10-YEAR MORTALITY IN A COMMUNITY- DWELLING POPULATION OF BRAZILIAN ELDERLY: THE BAMBUÍ COHORT STUDY OF AGEING}

doi:10.1136/jech.2011.1429760.12

\section{SP3-14 THE EFFECT OF OLDER EDUCATION ON KNOWLEDGE ABOUT HEALTHY LIFESTYLES: A RANDOMISED CONTROLLED TRIAL}

doi:10.1136/jech.2011.1429760.14

\begin{abstract}
1,2M F Lima-Costa, ${ }^{*}{ }^{1} \mathrm{~J} 0$ A Firmo, ${ }^{1,2}$ S V Peixoto, ${ }^{1} \mathrm{D}$ L Matos, ${ }^{1} \mathrm{E}$ Uchoa. ${ }^{1}$ Fundação Oswaldo Cruz, Belo Horizonte, Minas Gerais, Brazil; ${ }^{2}$ Universidade Federal de Minas Gerais, Belo Horizonte, Minas Gerais, Brazil
\end{abstract}

Introduction Predictors of mortality in old age have received much attention in the last 2 decades, but few studies have examined predictors of mortality in socioeconomically disadvantaged elderly populations in middle income countries. This study aimed at examining predictors of mortality in a community-dwelling population of Brazilian elderly with low income and very low schooling level.

Methods We used data of 1399 (80.3\% from total) residents aged 60 and over in Bambuí City, Brazil.

Results From 1997 to 2007, 599 participants died and 6.2\% were lost to follow-up, leading to 12415 person-years (pyrs) of observation. The death rate was 48.3 per 1000 pyrs. Age (Adjusted HR 1.40; 95\% CI 1.32 to 1.47 for each increment of 5 years), male gender (HR 1.80; 95\% CI 1.47 to 2.21 ), never married (HR $1.78 ; 95 \%$ CI 1.34 to 2.35 ) or a widow (HR 1.26; 95\% CI 1.03 to 1.56), poor self-rated health (HR 1.31; 95\% CI 1.02 to 1.69), inability to perform four or more Activities of Daily Living (HR 3.29; 95\% CI 2.17 to 5.00), number of cardiovascular risk factors (HR 1.51; 95\% CI 1.20 to 1.92 and $\mathrm{HR}=1.91 ; 95 \%$ CI 1.40 to 2.62 for two and three or more, respectively), Trypanosoma cruzi infection (HR 1.27; 95\% CI 1.06 to 1.52), and number of medications (HR 1.06; 95\% CI 1.02 to 1.20) were each significantly and independently associated with mortality. The Mini- Mental State Examination score showed a protective effect (HR 0.96; 95\% CI 0.93 to 0.99 for each increment of ten percentile unit).

Conclusions Except $T$ cruzi infection, other predictors of mortality were highly consistent with those found in more affluent elderly populations.

\section{SP3-13 PREVALENCE AND THE DETERMINANTS OF SMOKING AMONG HIGH-SCHOOL STUDENTS IN TIRANA, ALBANIA}

doi:10.1136/jech.2011.1429760.13

S Enkelejda.* Faculty of Nursing, Tirana, Albania

Introduction To assess the prevalence of smoking and its associated factors among high-school students in Tirana, Albania.

Methods An anonymous questionnaire survey was carried out among 797 high-school students (63\% females) in Tirana, the Albanian capital city, 2010. The questionnaire included sociodemographic data and information on smoking habits of students, their family members and their closed friends. Pearson's $\chi^{2}$ test was used to assess the difference in prevalence rates of smoking between students from different subgroups.

Results The overall prevalence of daily smoking was $14.9 \%(95 \% \mathrm{Cl}$ $12.5 \%$ to $17.6 \%$ ). It was $24.3 \%$ in males vs $9.4 \%$ in females $(\mathrm{p}<0.001)$. Other "predictors" of smoking were low academic performance $(p<0.001)$, having smoking friends $(p=0.001)$, and having smoking parents $(\mathrm{p}=0.001)$.

Conclusions Smoking habits among high-school students in Albania, resemble the patterns reported in other countries. Given the high smoking rate among adults, there is an urgent need to implement an effective tobacco control law in Albania.
M Taheri, ${ }^{*}$ A Amani, R Zahiri, M Mohammadi. Arak University of Medical Sciences, Arak, Iran

Methods This quasi-experimental study was performed in ten randomly selected villages (intervention $n=5$, control $n=5$ ) in rural Arak, Iran during 1389/2-1389/5. The healthy lifestyles were assessed using a multi-dimensional instrument designed for elderly persons. A self-designed questionnaire assessing the aims of the study (approved by a number of academic members) was also used. The intervention included: physical activity, advice on healthy food and other aspects of health lifestyle management. The aforementioned questionnaires were completed and the educational intervention actioned 3 month later. After the intervention data were collected again. The obtained data were analyse by SPSS software at the significant level of $\alpha=0.05$ with using $t$ test.

Results There were 408 elderly participants (intervention $n=212$, control $n=196)$ who participated in both baseline and post-intervention surveys. 221 (54.12\%) were male and 187 female (45.8\%). The mean age of participants was 71.3 years $(S D=9.9)$ and the majority were in the age range $60-65$ years (31.8\%). Many of the respondents were illiterate $(43.9 \%)$. The mean score of all parts of healthy lifestyles in experimental group increased significantly following the educational intervention. The older peoples mean knowledge grade increased from $28.6(\mathrm{SD}=7.4)$ to $35.9(\mathrm{SD}=6.8)$ $(p<0.001)$ following the education.

\section{SP3-15 THE HIV EPIDEMIC IN YUNNAN PROVINCE, CHINA, 1989-2007}

doi:10.1136/jech.2011.142976o.15

$\mathrm{L} L{ }^{*}{ }^{*} \mathrm{M}$ Jia, H Luo. Yunnan provincial centers of disease prevention and control, Kunming, Yunnan, China

Objective To investigate the characteristics and trends in the HIV epidemic in Yunnan province, China between 1989 and 2007.

Methods Statistical analysis of serological data from voluntary testing and counselling sites, medical case reports, mass screenings, sentinel surveillance, and other sources.

Results By 2007, a cumulative total of 57325 cases of HIV infection had been detected in Yunnan, and unsafe drug injection practices and unsafe sexual behaviours were identified as the dominant modes of transmission. Analysis shows HIV is spreading among IDUs, particularly among the Jingpo, Dai and Yi ethnicities, as well as among MSM and FSWs, some of whom also inject drugs. Rising numbers of reported HIV among male clients of FSWs (1.8\%), STD clinic patients $(2.1 \%)$, pregnant women $(0.50 \%)$, and blood donors $(0.084 \%)$ suggest infection in the general population is underway.

Conclusion The HIV epidemic in Yunnan has progressed to a concentrated epidemic. Future efforts must focus on not only on groups at risk for primary infection (IDUs, MSM, FSWs) but also on their lower-risk sexual partners. Future policy must improve efficacy and coverage of programs specifically targeting ethnic minorities, IDUs, MSM, and FSWs, particularly those who inject drugs or work at the street-level. 